Hierarchies of factor solutions in the intelligence domain: Applying methodology from personality psychology to gain insights into the nature of intelligence

\author{
Jonas W. B. Lang \\ Ghent University \\ Martin Kersting \\ University of Giessen \\ André Beauducel \\ University of Bonn
}




\begin{abstract}
Research on the structure of psychometric intelligence has used hierarchical models like the higher-order and the bi-factor model and has studied the hierarchical relationship between factors within these models. In contrast, research on the structure of personality has not only used hierarchical models but has also studied hierarchies of factor solutions. We clarify the theoretical and conceptual differences between hierarchical models and the solutions-hierarchy approach used in the field of personality research, and suggest that the solutions-hierarchy perspective can provide a novel perspective for intelligence research. We used the solutions-hierarchy approach to study four correlation matrices ( $N=230$ to $710 ; 38$ to 63 tests), and a large dataset $(N=$ 16,$823 ; 44$ tests). Results provided (a) insights into relationships between intelligence constructs across the hierarchy of factor solutions, and (b) evidence that intelligence has a 1-2-3-5 hierarchy of factor solutions with a $\mathrm{g}$ factor at the top, gc and gf factors at the second level, a speed-reasoning-knowledge taxonomy at the third level, and possibly a speed-reasoningfluency-knowledge-memory/perception taxonomy at the fifth level.
\end{abstract}

Keywords: Intelligence, cognitive abilities, hierarchies, structure, dimensionality 


\section{Hierarchies of Factor Solutions in the Intelligence Domain: Applying Methodology from Personality Psychology to Gain Insights Into the Nature of Intelligence}

Researchers have long been engaged in efforts to find taxonomies for the major domains of human individual differences. Although a consensus on the structure of a domain of individual differences is not necessarily a prerequisite for scientific progress, knowledge on the structure of a domain of individual differences is commonly helpful for integrating findings within a field and for developing a shared scientific language (e.g., Goldberg, 1993; Goldstein, Zedeck, \& Goldstein, 2002; John, Naumann \& Soto, 2008).

Research on the structure of psychometric intelligence started in the first half of the last century (e.g., Spearman, 1904, 1927; Thurstone, 1938a, 1938b). In the following decades, researchers developed a variety of different taxonomies (see Carroll, 1993, for an overview). Over time, most researchers reached a consensus on the idea that an optimal taxonomy for the intelligence domain should be a hierarchical structure with one or more broad abilities at the apex of the hierarchy and one or more levels of narrower abilities arranged below the broad abilities (Lubinski, 2004). This progress notwithstanding, research on the structure of psychometric intelligence is still an active field of research and researchers have continued to investigate the characteristics of the factors at each level of the hierarchy (Carroll, 2003; Goldstein et al., 2002; Johnson \& Bouchard, 2005; McGrew, 2009).

A notable difference between studies on the structure of intelligence and recent studies on the general structure of personality is that personality researchers frequently use some techniques and conceptualizations of hierarchy that have not yet been employed in intelligence research. Intelligence research typically relies on two types of hierarchical factor models: The higher-order model and the bi-factor (also known as the nested-factors and the hierarchical) model (e.g., Jensen \& Weng, 1994; Yung, Thissen, \& McLead, 1999). Personality researchers have also used 
these hierarchical models but have additionally used a conceptually different approach that focuses on studying hierarchies of factor solutions (Ashton, Lee, \& Goldberg, 2004; de Raad \& Barelds, 2008; Markon, 2009; Markon, Krueger, \& Watson, 2005; Saucier, 2009; Saucier \& Goldberg, 2001; Zuckerman, Kuhlman, \& Camac, 1988). This solutions-hierarchy approach entails a different conceptualization of hierarchy and is frequently used by personality researchers when the focus is on understanding and describing the structure of large datasets. The solutionshierarchy approach is also sometimes referred to as top-down factor analysis in the personality literature (Ashton, Lee, \& Goldberg, 2004; Goldberg, 2006; Waller, 2007).

In this article, we seek to build on personality research and suggest that the methodology frequently used in research on the structure of personality—studying hierarchies of factor solutions - also has implications for research on the structure of intelligence, and we believe that it is important to investigate these implications. We begin this article by clarifying the theoretical and conceptual differences between the hierarchical factor models commonly used in intelligence research, and the characteristics of solutions-hierarchy approach used in personality research. We follow up this conceptual section with analyses of five large datasets on intelligence using the solutions-hierarchy approach. Our article contributes to the literature by (a) clarifying different conceptualizations of hierarchy, by (b) establishing a conceptual link between research on the structure of personality and research on the structure of intelligence, and by (c) complementing existing studies and reviews on the structure of intelligence (e.g., Carroll, 1993).

\section{Extant Conceptualizations of Hierarchy in Intelligence Research}

\section{The Higher-Order Model}

One conceptualization of hierarchy that is frequently used in intelligence research is the higher-order model (e.g., Jensen \& Weng, 1994; Yung et al., 1999). Figure 1a shows a simple higher-order model. In this model, a broad second-order general factor influences three narrower 
abilities which in turn influence the measurement indicators or tests. One important assumption of this model is the idea that the second-order general factor causally influences the narrower abilities. Another characteristic assumption of the model is that the second-order general factor is not directly associated with the manifest tests or measurement indicators at the lowest level. The effect of the second-order general factor on the tests is mediated by the narrower (or first-order) factors. As a result, the second-order factor in the higher-order model has shared variance with the narrower abilities and this shared variance between the two is assigned to the higher-order factor as the causal source of this variance. The second-order factor also does not share variance with the measurement indicator (the test) that is not also shared between narrower abilities and the measurement indicator.

The higher-order model developed from scientific debate between Louis Thurstone (1939; Thurstone \& Thurstone, 1941) and Charles Spearman (1939). Spearman had long argued that the general factor extracted from a large intelligence test battery is a sort of mental energy that is responsible for correlations between tests (Spearman, 1904). In his original two-factor model, Spearman used only one latent variable for the general factor and suggested that the variance in each intelligence test consists of variance due to the general factor $g$ and a specific component that is unique to the specific test. Spearman's two-factor theory is similar to a one-factor model (Harman, 1976; Jensen \& Weng, 1994). Thurstone, in contrast, developed a multidimensional view of intelligence and preferred to extract oblique (correlated) factors from intelligence data. Spearman (1939) reanalyzed one of Thurstone's datasets using his two-factor theory. In this reanalysis, Spearman first reduced the number of indicators by aggregating tests that he considered to be similar and only then applied two-factor theory and concluded that the general factor from two-factor theory explained almost all of the correlations in the data between the test aggregates. This approach likely inspired Thurstone to develop the higher-order model by 
extracting a second-order factor from the correlations of his oblique factors in his later work (Thurstone \& Thurstone, 1941). The higher-order model can thus be seen as a compromise between Thurstone's work and Spearman's original idea that the shared variance between a battery of intelligence tests is caused by a general factor, or g (Jensen \& Weng, 1994; Lang, Kersting, Hülsheger, \& Lang, 2010).

A limitation of the higher-order model is the fact that the possible levels of hierarchy is restricted in practice. The reason is that higher-order factors are only extracted based on variance at the hierarchical level just below the level of interest (cf. Figure 1a). In practice, this characteristic of the model restricts the possible number of hierarchical structures. Carroll (1993), who conducted a large and comprehensive review of the intelligence literature using the higherorder model noted: "One is fortunate to obtain as many as three second-order factors, and this is the minimum number required to support an analysis for a single factor at the third order." (pp. 579).

\section{The Bi-Factor Model}

The bi-factor model (Holzinger \& Harman, 1938; Holzinger \& Swineford, 1937)—also known as the nested-factors model (Mulaik \& Quartetti, 1997) or the hierarchical model (Yung, Thissen, \& McLeod, 1999) —offers a second perspective on the status of broad and narrower abilities that is frequently employed in the intelligence literature. This bi-factor conceptualization is distinct the higher-order model and builds on the idea that broad and narrower abilities only differ in breadth and not in subordination (Humphreys, 1981). The term "bi-factor" derives from the idea that each measurement indicator (i.e., the tests) has more than one (typically two but more is possible, cf. Mulaik \& Quartetti, 1997) direct loadings — typically on a general and a narrow factor. Figure $1 \mathrm{~b}$ illustrates this conceptualization of the bi-factor model. Figure $1 \mathrm{~b}$ also shows that there is no correlation or direct path between the broad factor and the narrower 
abilities such that these factors are disjunct (i.e., orthogonal). Some methods employed in research using the bi-factor model explicitly require an orthogonal general factor (Jennrich \& Bentler, 2012). Other methods do not explicitly require the general factor to be orthogonal but in practice estimation with a general factor that is not orthogonal to other factors is difficult (Mulaik \& Quartetti, 1997; Rindskopf \& Rose, 1988) such that an orthogonal general factor is used in all research that we are aware of.

The fact that the hierarchical levels are disjunct in the model effectively divides the shared variance between the broad and the narrower factors to either the broad or the narrower factors such that not all shared variance between the general factor and the narrower abilities in the model is attributed to the general factor. The bi-factor perspective consequently does not make the assumption that the general factor has a causal effect on the narrower cognitive abilities. Instead, the broad and narrower factors differ only in breadth (and not in subordination like in the higher-order model). The general factor consequently has shared variance with the test itself that it does not also share with the narrower abilities.

A limitation of the bi-factor model is the fact that the model effectively eliminates shared variance between $g$ and the narrower-factors by orthogonalizing the levels. Removing shared variance through orthogonalization may frequently be desirable in applications (Carroll, 1993), and was especially desirable in studies on predictive validity before methodological techniques were developed that can incorporate shared variance between different levels of the hierarchy (Lang et al., 2010; Krumm, Schmidt-Atzert, \& Lipnevich, 2014). When researchers study the hierarchical structure of a construct, however, it may be theoretically desirable to use factor scores that reflects the nature of the data and theoretical assumptions. Orthogonalizing the levels then can be a theoretical limitation as shared variance between the different levels of the hierarchy is a core element of intelligence data (Jensen, 1998; Revelle \& Wilt, 2013). In studies 
on the hierarchical structure of intelligence, orthogonalizing the levels is also an empirical restriction as it effectively limits the number of possible levels to three (commonly only two; cf. Mulaik \& Quartetti, 1997).

\section{Hierarchies of Factor Solutions}

Research on hierarchies of factor solutions (Goldberg, 2006) is fundamentally based on exploratory factor analysis procedures with which most researchers are familiar. To derive a hierarchy of factor solutions, researchers start by extracting a solution with one (unrotated) factor and by calculating factor scores for this factor. In the next step, a two-factor solution is extracted and rotated, and factor scores as well as the correlations between these factor scores and the factor scores for the initial one factor solution are calculated. The procedure continues by extracting the rotated three factor solution, calculating factor scores for the three-factor solution, and by calculating correlations between the factor scores from the three-factor solution and the factor scores from the two factor solution. This routine continues for subsequent solutions with more factors.

As a refinement of the method, Waller (2007) has shown that it is possible to calculate the correlations between components or factors from different levels of the hierarchy without calculating the corresponding component scores or factor scores. Thereby, problems that might occur when factor scores are to be calculated (Beauducel, 2007) can be avoided (Waller, 2007).

In theory, a hierarchy of factor solutions allows for a number of factorial representations that is equal to the number of variables. However, because the major goal of factor analysis is to develop an adequate and parsimonious description of a domain of interest, the procedure will commonly stop much earlier.

One basic criterion for stopping is when no variables have their highest loadings on a factor. In this case, Goldberg (2006) advised researchers to stop at the level above that one. The 
reason for this recommendation is that factors without primary loadings are difficult to interpret and are commonly considered to be residual factors.

A second possible criterion for stopping is to rely on the standard approach used in exploratory factor analysis and examine the eigenvalue plot. In examining eigenvalue plots, the goal is to determine how many factors in a given covariance matrix are meaningfully different from random noise. The most common tools for evaluating eigenvalues are Cattell's graphical scree test (Cattell, 1966) and J. L. Horn's parallel analysis procedure (J. L. Horn, 1965b). The graphical scree test suggests that eigenvalue plots typically contain a marked decrease or cliff between the meaningful factors and the random factors. Horn's parallel analysis procedure simulates random eigenvalues using the properties of the data so that one can compare the actual eigenvalues with random eigenvalues.

A third criterion for stopping can be used when researchers simultaneously examine several datasets in the same research domain. A criterion for stopping in this context is when a solution does not replicate across datasets to a notable degree. Researchers can then confidentially assume that dataset-specific characteristics dominate in the factors solutions. For instance, research on the structure of personality has yielded no evidence that factors from solutions with more than six factors constantly replicate across languages and samples (e.g., Ashton, Lee, \& Goldberg, 2004; Ashton, Lee, Perugini et al., 2004). Most researchers studying several datasets in the same research domain examine eigenvalue plots as a first rough indication on the number of potentially meaningful factors in a dataset. The eigenvalue information, however, is rarely used to ultimately decide on the appropriateness of a taxonomy. For instance, in an analysis of 1,710 English personality adjectives or items, Ashton, Lee, and Goldberg (2004) decided to extract five or six factors even though the eigenvalue plot suggested the extraction of seven factors. Ashton, Lee, and Goldberg based this decision on the substantive interpretation of the solutions they extracted 
and the fact that solutions in other languages supported either five or six factors.

Hierarchies of factor solutions can be studied using either orthogonal or oblique rotations and either using principal components or factor analysis procedures (Goldberg, 2006; Waller, 2007). In the context of personality research, researchers commonly use principal components and orthogonal rotation procedures yielding uncorrelated components at each level of the hierarchy (Goldberg, 2006). The use of orthogonal rotations is based on the observation that many personality dimensions show only small intercorrelations. For the purpose of intelligence research, we suggest using oblique rotations because correlations between factors are likely important in intelligence research. There is strong evidence that intelligence measures are commonly correlated—frequently to a considerable degree (e.g., Jensen, 1998; Revelle \& Wilt, 2013). In addition, we propose that factor analysis instead of principal components is generally more appropriate because factor analysis takes measurement error into account (Gorsuch, 1983) and this may be relatively more important when indicators are correlated. Researchers have long used principal components instead of factor analysis for estimating hierarchies of factor solutions because the estimation of correlations between factors of solutions with different numbers of factors had long been problematic statistically (e.g. Grice, 2001). As already noted, Waller (2007) has recently solved this issue by showing how researchers can estimate the correlation matrix between the oblique latent factors for solutions at two different levels of a hierarchy of factor solutions using the rotation matrices for the two levels (see p. 749). Waller's procedure allows researchers to estimate the correlations between the true latent factors and does not require them to use approximations of the true correlation matrix calculated from factor scores (for principal components, the matrix estimated using the rotation matrices and Waller's procedure is identical to the matrix estimated using component scores cf. Grice, 2001).

There are important differences between the solutions-hierarchy approach and the two 
types of hierarchical models. A potential advantage of the solutions-hierarchy approach is that the extraction of factors at the different hierarchical levels is not influenced by decisions of the researcher regarding the extraction of factors at the other hierarchical levels. The reason is that the extraction of factors at each level is directly based on the manifest indicator variables (personality adjectives or personality-related statements in personality research and cognitive ability tests in cognitive ability research). In contrast, in the higher-order and bi-factor models, the extraction of broader factors is influenced by decisions on extraction of narrower factors. In the higher-order model, the factors higher in the hierarchy are based on the intercorrelations among the lower order factors and not directly on the manifest variables. Variance that the lowerorder factors do not capture can also not be captured by the higher-order factors in the model. In the bi-factor model the extraction of broad factors depends on decisions on the narrower factors because the variance in the manifest variables is distributed among the broad and the narrower factors. Consequently, when the number of narrow factors is reduced in a bi-factor model, some variance that has been represented by the narrow factors before can be represented by the broader factor (and vice versa). Figure 1c illustrates the nature of hierarchies of factor solutions. As shown in Figure 1c, all factors are directly estimated based on the measurement indicator variables. Furthermore, all factors are correlated with each other. Consequently, g and the narrower factors share variance with all other factors and the test itself.

In summary, the solutions-hierarchy approach has reached considerable sophistication and when used with oblique rotations it allows researchers to not only estimate a hierarchical structure with correlations between factors at different levels of the hierarchy but also between factors within each level of the hierarchy. The solutions-hierarchy approach avoids two specific restrictions of the hierarchical models typically used in intelligence research (orthogonal factors for the bi-factor, and the assumptions of causal higher-order factors in the higher-order model). 


\section{Intelligence Taxonomies and Previous Research Syntheses}

Existing syntheses of the intelligence literature have relied on higher-order approaches or bi-factor models (e.g., Carroll, 1982, 1993, 2003; French, Ekstrom, \& Price, 1963; McGrew, 2009). These overall literature reviews and other more specific studies have led to the development of a variety of intelligence taxonomies. To provide theoretical guidance for our investigation, we conducted a literature review of the cognitive ability literature that allowed us to develop hypotheses and expectations regarding the emergence of the broader factors at each level of the hierarchical structure extracted by the solutions-hierarchy method. The results of this literature review are shown in Table 1. Because we were primarily interested in parsimonious and replicable taxonomies that could be broadly applied to a variety of datasets, we focused our analyses to solutions with a limited number of factors that could realistically be expected to be replicable across different datasets and different sets of variables.

\section{The Present Investigation}

The overarching goal of the present investigation was to study the structure of intelligence using hierarchies of factor solutions. We focused on five datasets that were designed to cover a broad and representative range of ability measures. We then estimated hierarchies of factor solutions and studied which factors emerged at each level of the hierarchical structure of the solutions in each dataset. In so doing, we were interested (a) to what degree solutions in the datasets were similar to theoretical ideas in the reviewed cognitive ability literature and (b) to what degree solutions in different datasets were similar to each other. In addition to these research questions, we also studied how factors from solutions at different levels of the hierarchy (different numbers of factors) were related to each other.

\section{Method}




\section{Datasets}

We searched for datasets that had a sample size larger than 200, included a broad range of intelligence tests, and were originally assembled for the purpose of conducting an exploratory investigation of the general factor structure of intelligence (and not a specific domain of intelligence or cognitive abilities) in literature reviews (Carroll, 1993) and in databases of psychological literature (e.g., PsychINFO, Google Scholar, and WorldCat). This search yielded six datasets that fulfilled these criteria. For one dataset, no correlation matrices or raw data could be obtained (Jäger, 1967), and for one dataset only a correlation matrix of composite scores combining several cognitive abilities was available (Hakstian \& Cattell, 1974). For four datasets, a product-moment correlation or covariance matrix of the cognitive ability tests was available. These datasets were THUR41 (Thurstone \& Thurstone, 1941), HORN65 (J. L. Horn, 1965b), SCHO76 (Scholl, 1976), and WOTH (Wothke et al., 1990).

We additionally had access to an extended version of a previously published dataset (BEAU02; Beauducel \& Kersting, 2002). This extended dataset included the published data as well as additional data. The additional data included two additional tests, and 7,303 additional participants such that the study population included a total of 16,823 persons. The two additional tests were a dictation test assessing the ability to write down spoken text without orthographic errors, and a clerical work-sample test asking participants to determine postal rates based on a couple of different information parameters.

Table 2 provides details on the five datasets. We do not suggest that these datasets cover the universe of possible data sets. However, the datasets are a relevant subset of the relevant intelligence literature allowing for substantial investigation of the unrestricted nested-factors model. 


\section{Analytical Strategy and Statistical Analyses}

We examined the hierarchy of factor solutions using the procedures described in Goldberg (2006) and Waller (2007). As discussed previously, we relied on factor analysis and oblique rotation. Specifically, we used principal axis factor analysis. All solutions with more than one factor were promax-rotated $(m=3)$.

To study the relations between the solutions at the different levels of each dataset, we estimated the correlations between the factors using the procedures described in Waller (2007). We therefore relied on a modified version of Waller's (2007) syntax for the R programming environment (R Development Core Team, 2010). Waller's program was originally developed for orthogonal (varimax) rotation. We therefore modified the program for the use with promax rotation and factor analysis. Subsequently, we graphed the findings by translating the results of the solutions-hierarchy approach into a graph in the dot language (AT\&T Labs Research and Contributors, 2011).

We extracted solutions until the majority of the factors did not replicate across datasets anymore. Although we relied on the replication criterion, we also obtained eigenvalues for all five datasets as useful descriptive information on the number of potentially meaningful factors in the samples and as evidence on how dominant the first factor $(\mathrm{g})$ was in each of the matrices (see, e.g., Ashton et al., 2004). To foster the interpretation of the eigenvalues, we examined Cattell's graphical scree test (Cattell, 1966) and J. L. Horn's parallel analysis procedure (J. L. Horn, 1965b). The parallel analysis procedure simulates random eigenvalues using the properties of the data so that one can compare the actual eigenvalues with random eigenvalues.

\section{Results}

\section{Eigenvalues and Scree Plots}

Figure 2 provides eigenvalues and simulated random eigenvalues from the parallel analysis 
procedure for all five datasets. As indicated by Figure 2, there was evidence for a strong first factor in all eigenvalue plots.

The total number of factors to extract based on the graphical scree criterion (first marked eigenvalue increase) differed considerably across the five datasets. The scree criterion suggested that there were three or five factors in the THUR41 data, four factors in the HORN65 data, four factors in the SCHO76 data, two or six factors in the BEAU02 data, and two, three, or four factors in the WOTH90 data.

The number of extracted factors also differed when we used parallel analysis instead of the graphical scree procedure. As indicated by Figure 2, the simulated random eigenvalues derived by the parallel analysis procedure suggested that there were four factors in the THUR41, SCHO76, and WOTH90 datasets, three factors in the HORN65 data, and seven factors in the BEAU02 dataset.

\section{Hierarchy of Intelligence Factor Solutions}

Figure 3 to Figure 7 provide the graphical summaries of the solution-hierarchy analyses and show content descriptions as well as correlations between the factors at adjacent levels of the factor hierarchies for each dataset. In the graphs, the boxes represent the factors. The two-digit factor numbers refer to the overall number of factors in the solution from which the factor is derived (first digit), and the number of the factor in the solution (second digit). Correlations higher than .50 are shown with solid lines. For factors having no correlations higher than .50 to the next level, the highest correlation is shown using a dashed line.

First-Unrotated Factor. We started by examining the content of the first unrotated factors (FUF). These analyses revealed that tests with high loadings did not only included tests commonly considered to be good indicators of $g$ in the literature but also speed and fluency tests. The FUF was consequently quite diverse and broad in our datasets featuring large and diverse 
samples of cognitive ability tests.

Two-Factor Solutions. At the second level of hierarchy, three of the five datasets (THUR41, HORN65, and BEAU02) showed two factors which were reminiscent of a classic gfgc structure with a general crystallized factor (gc) capturing acquired skills, knowledge, and experience as well as a general fluid factor (gf) tapping logical thinking and problem-solving abilities in novel situations. The gf and gc factors all showed considerable correlations with the FUF or $g$ (see Figure 3 to Figure 7). In one dataset, gc had a stronger relation to $g$ than $g f$ (THUR41). In the two other datasets, gf was more strongly related to g than gc (HORN65, BEAU02). The relationships between $g$ and $g f$ as well as $g$ and $g c$ were frequently substantial but there was no correlation higher than $r=.74$.

The two datasets that did not clearly find a classic gf-gc structure showed different deviations from the gc-gf structure. In the $\mathrm{SCHO} 76$ data, the deviations from the gf-gc structure were relatively modest. In this dataset, verbal fluency tasks (Flu) combined with knowledgerelated tasks $(\mathrm{Kn})$ to form a strongly verbally-characterized gc factor. The assignment of verbalfluency tasks on gc has been proposed in the literature before. For instance, Carroll's threestratum theory (1993) conceptualizes verbal fluency as a subcomponent of gc. The underlying idea is that verbal fluency tasks commonly require that person's retrieve a substantive variety of vocabulary from (crystallized) long-term memory. However, in the SCHO76 data, also fluency tasks that require only very basic processing of verbal material load on the gc factor so that the combined factor in this datasets is broader than the gc factor expected by gf-gc theory and its modifications (e.g., Hakstian \& Cattell, 1974; J. L. Horn \& Cattell, 1966).

The deviations from the gc-gf structure in the other dataset, the WOTH90 data, were more substantial. In this dataset, one factor captured all types of strongly speeded tasks including simple speeded fluency tests (Spe-Flu), and the other factor included all less speeded reasoning 
(Re) and knowledge (Kn) tasks. This structure is most reminiscent of Ackerman's (1988) differentiation between level/power and speed abilities in his extended version of Guttmann's radex model (Guttmann, 1965). Overall, it should be noted that there are substantial differences between the gc-gc factors in the different data sets and that also the correlation of gf, gc, and $g$ shows considerable variation across data sets.

Three-Factor Solutions. There was considerable agreement on the third level of the hierarchy. In all five datasets, a basic three-factor structure emerged that consisted of one factor with basic speed abilities (Spe), one factor with reasoning tasks (Re), and one factor with knowledge tasks $(\mathrm{Kn})$. The speed factor was characterized by tasks that require people to perform simple overlearned operations quickly. The reasoning factor was characterized by tasks that require the deep processing of complex figural, verbal, and numerical tasks. Finally, the knowledge factor contained tasks that asked for factual knowledge from long-term memory and was very closely related to the gc factors from the two-factor solutions but did not contain verbal tasks that also required reasoning. Although we found this basic structure in all five datasets, there were nevertheless differences in the exact nature of these factors. Especially, the datasets differed in how fluency (Flu), memory (Me), and perceptual speed tasks (Perc) were assigned to the three factors.

Four-Factor Solutions. At the fourth level, the speed-reasoning-knowledge taxonomy from the third level remained intact in all solutions. The new fourth factor that emerged differed between the datasets. In three datasets (HORN65, THUR41, and WOTH90), a new separate fluency factor emerged. In the other two datasets (SCHO76, and BEAU02), the four-factor solution included a separate memory factor in addition to the speed-reasoning-knowledge taxonomy.

Five-Factor Solutions. At the fifth level, four datasets included four core factors: speed, 
reasoning, knowledge, and fluency. The exception was the SCHO76 data in which knowledge and fluency could not be separated. In addition, there was evidence for a memory-perception factor. The empirical picture this factor was more difficult to interpret because not all datasets included a considerable range of memory and perception tasks. In two datasets (SCHO76, BEAU02), the memory factors from the four-factor solutions continued to exist. These datasets contained a range of memory tasks but not prototypical perceptual tasks. The perceptual tasks in these datasets also involved either speed or reasoning and consequently loaded on these factors. In one dataset (THUR41), a perception factor emerged. This dataset included a limited number of memory tasks but a considerable range of perceptual tasks that were not primarily characterized by speed or reasoning. Finally, in the only dataset containing both a considerable range of memory and perception tasks, we found a combined perception-memory factor at the fifth level (WOTH90).

Six, Seven, and Eight Factors. While the present set of analyses suggests that there is considerable agreement at the first five levels of the solution hierarchy, we found considerably less convergence at subsequent levels and a variety of specific factors. These factors included combinations of naming speed (Nam-Spe), calculation (Cal), 2D rotation (2D-Rot), visual rotation in general (VisRot), counting (Cou), word fluency (WoFlu), ideational fluency (IdeFlu), general ideational (Id), and mathematical (Math) abilities.

The only finding that occurred in more than one dataset was that the reasoning factor collapsed into content-material specific subfactors. This split-up occurred in three of the five datasets (THUR41, SCHO76, and BEAU02). In two of these three datasets (SCHO76 and BEAU02), these content-specific reasoning factors largely represented verbal, numerical, and figural material and were in correspondence with the figural-verbal-numerical taxonomy (e.g., Ackerman, 1988; Guilford, 1967). 


\section{Discussion}

In this article, we built on personality research and suggested that studying hierarchies of factor solutions can also be useful for research on the structure of intelligence. Our reanalysis yielded new insights into the nature of the intelligence construct by identifying some structural consistencies at the top of the hierarchy of factor solutions that were reasonably stable across the five datasets. In this discussion section, we elaborate both on the substantive findings of our investigation as well as the methodological implications of studying hierarchies of factor solutions in intelligence research. We begin by discussing the substantive findings.

\section{Hierarchies of Intelligence Factor Solutions}

The present investigation focused on five datasets on the general structure of intelligence. Figure 8 provides a summary of our findings at the first five level of the hierarchy of factor solutions in these five datasets.

As Figure 8 illustrates, one finding of our investigation was that we found evidence for the gc-gf distinction proposed by Cattell and J. L. Horn (Cattell, 1943b, 1963; J. L. Horn, 1965a, 1976) at the second level of the hierarchy. A gf factor emerged in four, and a gc factor emerged in three of the five datasets. There were some differences in the relations between the gc and gf factors at the second level and the $\mathrm{g}$ factor. Although one of these two relations was typically high in each of the datasets, none of these correlations exceeded $r=.74$. Our analyses consequently suggest that both gc and gf carry meaning that is different from the gactor at the top in hierarchies of factor solutions.

A second major finding was that there was a factor structure with a reasoning, a speed, and a knowledge factor at the third level of the solutions hierarchy. This basic structure was present in all five datasets (see Figure 8) even though the exact nature of the factors somewhat differed across the five datasets. The fact that we found this basic taxonomy in all five datasets is 
remarkable given that the datasets considerably varied in the used material, context of the data collection (personnel selection vs. participation in research), time (1940s to the 2000s), and sample characteristics. The reasoning-speed-knowledge taxonomy is not identical to a specific model in the previous intelligence literature but nevertheless shows considerable similarities to models previously discussed in the literature. Specifically, the distinction between speed and reasoning abilities can also be found in the radex model of intelligence (e.g., Marshalek et al., 1983). However, both speed and knowledge tasks can be classified into the rule-application segment of the radex model (Marshalek et al., 1983). Moreover, gc has been located in the ruleapplication segment of the radex model (Marshalek et al., 1983), so that the radex model does not distinguish between gc, knowledge and speed. It should be noted that the radex model could only be calculated by means of smallest space analysis (Guttman, 1968), which leads to a representation of the data that can usually not be found by means of factor analysis. Furthermore, the distinction between reasoning and speed abilities is also a key building block of the Berlin model of Intelligence structure (Beauducel \& Kersting, 2002; Jäger et al., 1997; Schulze, 2005; Süß \& Beauducel, 2005). However, this model does not consider knowledge abilities. The reason for the fact that we did not finding content factors for verbal, numerical, and figural abilities at the third level that have been found in other models (Ackerman, 1988; Jäger et al., 1997; Marshalek et al., 1983) could also be related to the fact that the solutions-hierarchy approach aims at finding the most robust structures in the data, whereas smallest space analysis and related faceted confirmatory factor models (Süß \& Beauducel, 2005) are aimed to identify complex overlapping structures.

The third major finding was that we found considerable agreement on the fourth and fifth level of the hierarchy (see Figure 8). Specifically, we found evidence for a taxonomy consisting of factors for speed ( 5 of 5 datasets), reasoning ( 5 of 5 datasets), fluency ( 4 of 5 datasets), and 
knowledge (4 of 5 datasets). In addition to this speed-reasoning-fluency-knowledge taxonomy, there was also some evidence for the existence of a memory-perception factor (3 datasets). We speculate that a problem for identifying this factor was that some of the datasets did not contain a sufficient number of both prototypical perception and prototypical memory tasks. Accordingly, a factor primarily characterized by memory tasks emerged in two datasets (SCHO76, BEAU02), and a perception factor emerged in one dataset with a small number of memory tests (THUR41). In the dataset that features a variety of both tasks (WOTH90), an integrated factor emerged.

In summary, this study suggests that intelligence as operationalized in the five datasets that we studied can be characterized by a 1-2-3-5 hierarchy of factor solutions with a $\mathrm{g}$ factor at the top of the solutions hierarchy, gc and gf factors at the second level, a speed-reasoning-knowledge taxonomy at the third level, and a four- or five-factor taxonomy with a speed, a reasoning, a fluency, a knowledge and possibly a memory-perception factor at the fourth or fifth level. The evidence for the speed-reasoning-knowledge taxonomy at the third level was somewhat stronger than the evidence for the gc/gf and the speed-reasoning-fluency-knowledge-memory/perception taxonomy at the fifth level because we found evidence for factors of this type in all five datasets (see Figure 8). Overall, the findings of this study suggest that intelligence has a relatively stable hierarchy of factor solutions in the five datasets we investigated. The differences between the hierarchies of factor solutions in the five datasets were overall comparable to the differences typically found between investigations in the field of personality. Lexical studies of personality commonly also show considerable agreement but nevertheless also show some deviations across different languages or different sets of items (Ashton, Lee, \& Goldberg, 2004; Ashton, Lee, Perugini et al., 2004).

Our study provides researchers, test developers, and practitioners with a different representation of the structure of intelligence. This different representation has several 
implications for intelligence research.

One implication of our study is that its findings provide a basis for structuring future metaanalyses and reanalyses of intelligence research just like lexical studies studying hierarchies of factor solutions and the five- and six-factor taxonomies were useful for personality researchers (Ashton \& Lee, 2005; Saucier \& Goldberg, 2001). The 1-2-3-5 taxonomy includes relatively broad factors and can thus be used on datasets that use a variety of different types of intelligence batteries. This characteristic may especially be useful when researchers seek to structure literature on the relationship between different types of intelligence measures and outcome criteria.

A second implication of our study for intelligence research is that it could provide a basis for the development of new intelligence batteries and measures. One possibility would be to develop a battery that captures the entire 1-2-3-5 structure. However, because hierarchies of factor solutions typically include considerable overlap between adjacent levels, we believe that a convenient approach for researchers could be to target one or two specific levels of the 1-2-3-5 taxonomy. Researchers could then try to select a set of intelligence tasks that adequately capture these levels of the taxonomy (e.g., the third or the second and the fifth level). An advantage of this approach is that it is relatively simple. Consequently, there may be a good chance that factor solutions replicate well across different contexts and datasets.

\section{Methodological Implications}

Intelligence research has traditionally focused on higher-order factor analysis and bi-factor analysis, and these approaches have contributed to significant progress in the field of intelligence research. A limitation of these approaches is that the extraction of factors at each level is affected by the extraction of factors at other levels. This characteristic limits the number of hierarchical levels in higher-order and bi-factor models to commonly not more than three (cf. Carroll, 1993; Mulaik \& Quartetti, 1997). The solutions-hierarchy approach adds a different representation of 
intelligence. Researchers can use the solutions-hierarchy approach to study relationships between a large number of levels and to graph relationships between closely related factors. The solutionshierarchy approach may especially be useful when researchers are interested in providing a description of an intelligence dataset and when they seek to identify differences and similarities across datasets from different traditions of intelligence research.

Another way in which the solutions-hierarchy approach may be useful for researchers is when they seek to study correlated factors with different levels of broadness/generality in outcome criteria but do not want to make assumptions on the causal direction of the correlations (Humphreys, 1981; Sternberg, 1981). The unspecified causal direction between the more general and more specific factors in the solutions-hierarchy approach may have practical implications when it comes to studying the role of correlated intelligence constructs in outcome criteria (Lang et al., 2010; Krumm et al., 2014). When researchers assume that a $\mathrm{g}$ factor causes the correlations between intelligence tests, the higher-order model and incremental validity analysis are the adequate tools for studying the role of $\mathrm{g}$ and narrower intelligence constructs in outcome criteria. In the higher-order model, $g$ has a causal influence on the narrower cognitive abilities (see Figure 1a) and the higher-order model therefore assumes that all shared variance between $g$ and the narrower cognitive abilities is caused by $\mathrm{g}$. In line with this idea, $\mathrm{g}$ should be entered first in incremental validity analyses. In contrast, when researchers wish to not make the a-priori assumption that shared variance results from $g$, they can base their research on the bi-factor or the solutions-hierarchy approach as both approaches do not make assumptions on the causal order of $\mathrm{g}$ and the narrower cognitive abilities. In the solutions-hierarchy approach, shared variance between $g$ and the narrower cognitive abilities can be retained without assumptions on the source of the shared variance. The relationship between the correlated factors from a hierarchy of factor solutions and the criterion can then be studied using regression-based techniques that do not use 
assumptions on a causal order of the predictors (Lang et al., 2010). Regression-based techniques of this type include relative importance analysis (Grömping, 2007) and dominance analysis (Azen \& Budescu, 2003). Studying hierarchies of factor solutions consequently does not only provide a different approach for studying the structure of intelligence but also has implications for studying relationships between intelligence constructs and outcome criteria.

\section{Limitations}

One limitation of our study concerns the source of the differences between datasets. As noted previously, there were considerable similarities in the factor structures from each of the datasets, and these similarities were surprisingly large given that the five datasets considerably varied in the used material, the context (applicants vs. volunteers), the time (1940s to the 2000s), and the characteristics of the participants. Nevertheless, there were also some relevant differences between the datasets. A limitation of our investigation is that it is difficult to tell why these differences occurred and what factor likely caused these differences.

A second limitation is the number of manifest variables in the current analyses. The number of tests in the five datasets ranged from 38 to 63 . Although these numbers are considerably higher than the number of tests in most investigations on the structure of intelligence and are the largest available matrices on the general structure of intelligence, these numbers are still considerably smaller than the number of manifest variables in studies examining hierarchies of factor solutions in the field of personality research. The reason is that gathering data on a manifest variable in personality research (a personality item consisting of a Likert-scale rating of a personality-related adjective or statement) consumes less time than gathering data on a manifest variable in intelligence research (a test consisting of multiple similar items). Nevertheless, the factors in the stable taxonomies we identified still were all based on a considerable number of variables. Furthermore, it is likely that the variables in intelligence-structure investigations are 
considerably more reliable than the single-item ratings used in personality research so that the higher reliability of the variables may partly make up for the lower overall number of variables.

Another limitation of our reanalyses is the fact that the datasets in our investigation consisted of relatively young samples. The intelligence literature suggests that gc and gf develop differently across the lifespan (Cattell, 1963; Horn, 1976) and also that performance in specific tests changes differently across the life span and across cohorts (Skirbekk, Stonawski, Bonsang, $\&$ Staudinger, 2013). We accordingly recommend future research using older and more agediverse samples.

In addition to the described limitations that relate to the nature of the datasets that we reanalyzed, there are also limitations of the solutions-hierarchy approach method. One limitation of the approach is that it is based on exploratory factor analysis. Exploratory factor analysis is data-driven in its nature and does not directly allow researchers to test the degree to which prespecified factor models fit a particular dataset.

A second limitation of the solutions-hierarchy approach is that it is typically difficult to objectively judge how many factors should be extracted. Decisions on the number of factors that should be extracted typically depends on the interpretation of the factors especially when researchers study different datasets. This limitation is not specific to the use of hierarchies of factor solutions for the purpose of studying the structure of intelligence.

A third limitation of the solutions-hierarchy approach is that it is primarily suited to describe relationships between intelligence factors. The solutions-hierarchy approach is not designed for testing specific causal theories on how intelligence factor influence each other. For instance, the solutions-hierarchy approach is capable of describing the correlation of $g$ with gc and gf factors at the second level. However, the approach is not capable of, for instance, testing theoretical ideas on effects of $g$ on gc and gf (Undheim \& Gustaffson, 1987; Valentin Kvist \& 
Gustafsson, 2008).

Finally, a fourth limitation of the solutions-hierarchy and most other research on the structure of intelligence is the fact that factor models do not necessarily provide insights on the functional level of the human brain (Bartholomew, 2004). In an ideal scenario, researchers would like to find correspondence between intelligence factors identified in research on the structure of intelligence and individual differences in biological characteristics. However, correspondences of this type are not easy to identify and require biological research (Bartholomew, 2004).

\section{Future Directions}

Although our investigation was based on five large datasets, it nevertheless seems necessary and worthwhile to further investigate the structure of intelligence using the solutionshierarchy approach by conducting new research and by applying the approach more broadly in other intelligence variable sets and samples. For instance, future research could further investigate the memory/perception factor. One possible approach for doing so would be to combine perception tasks from the WOTH90 and THUR41 datasets with the memory tasks from BEAU02 and WOTH90 in one data collection. Another important goal would be to investigate older samples. As we noted in the limitations section, most of the datasets in our investigation consisted of relatively young samples and the structure of intelligence may be subject to change across the lifespan.

The development of models of intelligence has often been based on new methods of multivariate data analysis, especially in the domain of factor analysis. Examples include Spearman's two-factor theory and Thurstone's primary mental abilities. The present study is just another example for the close relationship between multivariate data analysis and models of intelligence. It might therefore be expected that further developments in the domain of factor analysis will further our understanding of intelligence. Since the solutions-hierarchy approach 
does not identify the hierarchical level of the factors with the causal hierarchy of the factors, it might be conceived to specify causal relations in a second step. This would allow for a specification of causal relations across all levels of the hierarchy (upwards, on the same level, and downwards) according to theoretical assumptions and not simply according to the hierarchical level of the factors.

\section{Conclusion}

In this article, we have suggested that studying hierarchies of factor solutions can provide novel insights into the structure of psychometric intelligence. In our reanalysis of five datasets, we applied the modified solutions-hierarchy approach to five large scale investigations on the structure of intelligence. Our analyses suggest that intelligence has a 1-2-3-5 hierarchy of factor solutions with a g factor at the top of the hierarchy, gc and gf factors at the second level, a speedreasoning-knowledge taxonomy at the third level, and a four- or five-factor taxonomy with a speed, a reasoning, a fluency, a knowledge and possibly a memory-perception factor at the fourth or fifth level. We believe that these findings provide a building block for future research on intelligence using the solutions-hierarchy approach and have the potential to contribute to a better understanding of the structure of intelligence. 


\section{References}

Ackerman, P. L. (1988). Determinants of individual differences during skill acquisition: Cognitive abilities and information processing. Journal of Experimental Psychology: General, 117, 288-318. doi: 10.1037/0096-3445.117.3.288

Ackerman, P. L., Beier, M. E., \& Boyle, M. O. (2005). Working memory and intelligence: The same or different constructs? Psychological Bulletin, 131, 30-60. doi: 10.1037/00332909.131.1.30

Azen, R., \& Budescu, D. V. (2003). The dominance analysis approach for comparing predictors in multiple regression. Psychological Methods, 8, 129-148. doi: 10.1037/1082$989 X .8 .2 .129$

Amthauer, R. (1955). Handanweisung für den Intelligenz - Struktur - Test (I-S-T). [Manual for the Intelligence - Structure - Test (I-S-T)], Göttingen, Germany: Hogrefe

Ashton, M. C., \& Lee, K. (2005). A defence of the lexical approach to the study of personality structure. European Journal of Personality, 19, 5-24. doi: 10.1002/per.541

Ashton, M. C., Lee, K., \& Goldberg, L. R. (2004). A hierarchical analysis of 1,710 English personality-descriptive adjectives. Journal of Personality and Social Psychology, 87, $707-$ 721. doi: $10.1037 / 0022-3514.87 .5 .707$

Ashton, M. C., Lee, K., Perugini, M., Szarota, P., de Vries, R. E., Di Blas, L., et al. (2004). A six factor structure of personality descriptive adjectives: Solutions from psycholexical studies in seven languages. Journal of Personality and Social Psychology, 86, 356-366. doi: $10.1037 / 0022-3514.86 .2 .356$

Ashton, M. C., Lee, K., \& Vernon, P. A. (2001). Which the real intelligence? A reply to Robinson (1999). Personality and Individual Differences, 30, 1353-1359. doi: $10.1016 / \mathrm{S} 0191-8869(00) 00117-3$ 
Ashton, M. C., Lee, K., \& Vernon, P. A. (2005). Gc and Gf are both valid intelligence factors: Commentary on Robinson (2005). Personality and Individual Differences, 39, 999-1004. doi: 10.1016/j.paid.2005.03.019

AT\&T Labs Research and Contributors (2011). Graphviz (Version 2.28.0) [Software]. Florham Park, NJ: Author.

Bartholomew, D. J. (2004). Measuring intelligence: Facts and fallacies. Cambridge, UK: Cambridge University Press.

Beauducel, A. (2007). In spite of indeterminacy many common factor score estimates yield an identical reproduced covariance matrix. Psychometrika, 72, 437-441. doi: 10.1007/s11336$005-1467-5$

Beauducel, A., \& Kersting, M. (2002). Fluid and crystallized intelligence and the Berlin Model of Intelligence Structure (BIS). European Journal of Psychological Assessment, 18, 97-112. doi: $10.1027 / 1015-5759.18 .2 .97$

Botzum, W. A. (1951). A factorial study of the reasoning and closure factors. Psychometrika, 16, 361-386. doi: 10.1007/BF02288801

Carroll, J. B. (1982). The measurement of intelligence. In R. Steinberg (Ed.), Handbook of human intelligence (pp. 29-120). London, UK: Cambridge University Press.

Carroll, J. B. (1993). Human cognitive abilities: A survey of factor-analytic studies. Cambridge, UK: Cambridge University Press.

Carroll, J. B. (2003). The higher-stratum structure of cognitive abilities: Current evidence supports $g$ and about ten broad factors. In H. Nyborg (Ed.). The scientific study of general intelligence: Tribute to Arthur R. Jensen (pp. 1-22). Oxford, UK: Elsevier.

Cattell, R. B. (1943b). The measurement of adult intelligence. Psychological Bulletin, 40, 153193. doi: $10.1037 / \mathrm{h} 0059973$ 
Cattell, R. B. (1963). Theory of fluid and crystallized intelligence: A critical experiment. Journal of Educational Psychology, 54, 1-22. doi: 10.1037/h0046743

Cattell, R. B. (1966). The scree test for the number of factors. Multivariate Behavioral Research, 1, 245-276. doi: 10.1207/s15327906mbr0102_10

de Jong, P. F., \& Das-Smaal, E. A. (1995). Attention and intelligence: The validity of the star counting test. Journal of Educational Psychology, 87, 80-92. doi: 10.1037/00220663.87 .1 .80

de Raad, B., \& Barelds, D. P. H. (2008). A new taxonomy of Dutch personality traits based on a comprehensive and unrestricted list of descriptors. Journal of Personality and Social Psychology, 94, 347-364. doi: 10.1037/0022-3514.94.2.347

Ekstrom, R.B., French, J. W., Harman, H. H., \& Derman, D. (1976). Manual for kit of factorreferenced cognitive tests. Princeton, NJ: Educational Testing Service.

French, J. W., Ekstrom, R. B., \& Price, L. A. (1963). Manual for kit of reference tests for cognitive factors. Princeton, NJ: Educational Testing Service.

Goldberg, L. R. (1993). The structure of phenotypic personality traits. American Psychologist, 48, 26-34. doi: 10.1037/0003-066X.48.1.26

Goldberg, L. R. (2006). Doing it all Bass-Ackwards: The development of hierarchical factor structures from top down. Journal of Research in Personality, 40, 347-358. doi:10.1016/j.jrp.2006.01.001

Goldstein, H. W., Zedeck, S., \& Goldstein, I. L. (2002). g: Is this your final answer? Human Performance, 15, 123-142. doi: 10.1207/S15327043HUP1501\&02_08

Gorsuch, R. L. (1983). Factor analysis (2 ${ }^{\text {nd }}$ ed.). Hillsdale, NJ: Erlbaum.

Grice, J. W. (2001). Computing and evaluating factor scores. Psychological Methods, 6, 430450. doi: 10.1037/1082-989X.6.4.430 
Grömping, U. (2007). Estimators of relative importance in linear regression based on variance decomposition. The American Statistician, 61, 139-147. doi:10.1198/000313007X188252

Guilford, J. P. (1967). The nature of human intelligence. New York, NY: McGraw-Hill.

Gustafsson, J.-E. (1984). A unifying model for the structure of intellectual abilities. Intelligence, 8, 179-203. doi: 10.1016/0160-2896(84)90008-4

Guttman, L. (1965). The structure of the interrelations among intelligence tests. In C. W. Harris (Eds.), Proceedings of the 1964 Invitational Conference on Testing Problems. (pp. 33-44) Princeton, NJ: Educational Testing Service. Retrieved from http://www.eric.ed.gov

Guttman, L. (1968). A general nonmetric technique for finding the smallest coordinate space for a configuration of points. Psychometrika, 33, 469-506.

Hakstian, A. R., \& Cattell, R. B. (1974). The checking of primary ability structure on a broader basis of performances. British Journal of Educational Psychology, 44, 140-154.

Harman, H. H. (1976). Modern factor analysis ( $3^{\text {rd }}$ ed.). Chicago, IL: University of Chicago Press.

Holzinger, K. J., \& Harman, H. H. (1938). Comparison of two factorial analyses. Psychometrika, 1, 45-60. doi: 10.1007/BF02287919

Holzinger, K. J., Swineford, F. (1937). The bi-factor method. Psychometrika, 2, 41-54. doi: 10.1007/BF02287965

Horn, J. L. (1965a). Fluid and crystallized intelligence: A factor analytic study of the structure among primary mental abilities. Unpublished doctoral dissertation, University of Illinois. ,

Horn, J. L. (1965b). A rationale and test for the number of factors in factor analysis. Psychometrika, 30, 179-185. doi: 10.1007/BF02289447

Horn, J. L. (1976). Human abilities: A review of research and theory in the early 1970s. Annual Review of Psychology, 27, 437-485. doi: 10.1146/annurev.ps.27.020176.002253 
Horn, J. L., \& Cattell, R. B. (1966). Refinement and test of the theory of fluid and crystallized general intelligences. Journal of Educational Psychology, 57, 253-270. doi: $10.1037 / \mathrm{h} 0023816$

Horn, W. (1962). Handanweisung für das Leistungsprüfsystem (L-P-S). [Manual for the achievement test system]. Göttingen, Germany: Hogrefe.

Humphreys, L. G. (1981). The primary mental ability. In M. P. Friedman, J. R Das, \& N. O'Connor (Eds.): Intelligence and learning (pp. 87-102). New York: Plenum.

Jäger, A. O. (1967). Dimensionen der Intelligenz. [Dimensions of intelligence]. Göttingen, Germany: Hogrefe

Jäger, A. O., Süß, H.-M. \& Beauducel, A. (1997). Berliner Intelligenzstruktur - Test. Form 4. [Berlin intelligence structure test. Form 4.]. Göttingen, Germany: Hogrefe

Jennrich, R. I., \& Bentler, P. M. (2012). Exploratory bi-factor analysis: The oblique case. Psychometrika, 77, 442-454. doi: 10.1007/S11336-012-9269-1

Jensen, A. R. (1998). The g factor. Westport, CT: Praeger.

Jensen, A. R., \& Weng, L.-J. (1994). What is a good g? [Editorial]. Intelligence, 18, 231-258. doi: 10.1016/0160-2896(94)90029-9

John, O. P., Naumann, L. P., \& Soto, C. J. (2008). Paradigm shift to the integrative big-five trait taxonomy: History, measurement, and conceptual issues. In O. P. John, R. W. Robins, \& L. A. Pervin (Eds.), Handbook of personality: Theory and research (pp. 114-158). New York, NY: Guilford Press.

Johnson, W. \& Bouchard, T. J., Jr. (2005). The structure of human intelligence: It is verbal, perceptual, and image rotation (VPR), not fluid and crystallized. Intelligence, 33, 393-416. doi: 10.1016/j.intell.2004.12.002

Krumm, S., Schmidt-Atzert, L., \& Lipnevich, A. A. (2014). Specific cognitive abilities at work: 
A brief summary from two perspectives. Journal of Personnel Psychology, 13, 117-122. doi: $10.1027 / 1866-5888 / \mathrm{a} 000117$

Lang, J. W. B., Kersting, M., Hülsheger, U. R., \& Lang, J. (2010). General mental ability, narrower cognitive abilities, and job performance: The perspective of the nested-factors model of cognitive abilities. Personnel Psychology, 63, 595-640. doi: 10.1111/j.1744$6570.2010 .01182 . x$

Lubinski, D. (2004). Introduction to the special section on cognitive abilities: 100 years after Spearman's (1904) "'General intelligence,' objectively determined and measured." Journal of Personality and Social Psychology, 86, 96-111. doi: 10.1037/0022-3514.86.1.96

Markon, K. E., Krueger, R. F., \& Watson, D. (2005). Delineating the structure of normal and abnormal personality: An integrative hierarchical approach. Journal of Personality and Social Psychology, 88, 139-157. doi: 10.1037/0022-3514.88.1.139

Marshalek, B., Lohman, D. F., \& Snow, R. E. (1983). The complexity continuum in the radex and hierarchical models of intelligence. Intelligence, 7, 107-127. doi: 10.1016/01602896(83)90023-5

McGrew, K. S. (2009). CHC theory and the human cognitive abilities project: Standing on the shoulders of the giants of psychometric intelligence research. Intelligence, 37, 1-10. doi:10.1016/j.intell.2008.08.004

Mulaik, S. A., \& Quartetti, D. A. (1997). First order or higher order general factor? Structural Equation Modeling, 4, 193-211. doi: 10.1080/10705519709540071.

R Development Core Team. (2010). R: A language and environment for statistical computing (Version 2.12.0) [Computer software]. Vienna: R Foundation for Statistical Computing. Retrieved from http: //cran.r-project.org/.

Revelle, W., \& Wilt, J. (2013). The general factor of personality: A general critique. Journal of 
Research in Personality, 47, 493-504. doi: 10.1016/j.jrp.2013.04.012

Rindskopf, D., \& Rose, T. (1988). Some theory and applications of confirmatory second-order factor analysis. Multivariate Behavioral Research, 23, 51-67. doi:

10.1207/s15327906mbr2301_3

Saucier, G. (2009). Recurrent personality dimensions in inclusive lexical studies: Indications for a big six structure. Journal of Personality, 77, 1577-1613. DOI: 10.1111/j.14676494.2009.00593.x

Saucier, G., \& Goldberg, L. R. (2001). Lexical studies of indigenous personality factors: Premises, products, and prospects. Journal of Personality, 69, 847-879. doi: 10.1111/14676494.696167

Scholl, R. (1976). Eine empirische Untersuchung zur Faktorenstruktur der Intelligenz. [An empirical investigation oft he factor-structure of intelligence]. Hamburg, Germany: Lüdke.

Schulze, R. (2005). Modeling structures of intelligence. In O. Wilhelm \& R.W. Engle (Eds.), Handbook of understanding and measuring intelligence (pp. 241-263). London: Sage.

Schweizer, K., Moosbrugger, H., \& Goldhammer, F. (2005). The structure of the relationship between attention and intelligence. Intelligence, 33, 589-611. doi:

10.1016/j.intell.2005.07.001

Skirbekk, V., Stonawski, M., Bonsang, E., \& Staudinger, U. M. (2013). The Flynn effect and population aging. Intelligence, 41, 169-177. doi: 10.1016/j.intell.2013.02.001

Spearman, C. (1904). "General intelligence," objectively determined and measured. American Journal of Psychology, 15, 201-292. DOI: 10.1037/11491-006

Spearman, C. (1927). The abilities of man, their nature and measurement. New York, NY: Macmillan.

Spearman, C. (1939). Thurstone's work re-worked. Journal of Educational Psychology, 30, 1-16. 
doi: $\underline{10.1037 / \mathrm{h} 0061267}$

Sternberg, R. (1981). The evolution of theories of intelligence. Intelligence, 5, 209-230. doi: $10.1016 / \mathrm{S} 0160-2896(81) 80009-8$

Süß, H.-M. \& Beauducel, A. (2005). Faceted models of intelligence. In O. Wilhelm \& R. W. Engle (Eds.), Handbook of understanding and measuring intelligence (pp. 313-332). London, UK: Sage.

Taylor, C. W. (1947). A factorial study of fluency in writing. Psychometrika, 12, 239-262. doi: 10.1007/BF02288939

Thurstone, L. L. (1938a). Primary Mental Abilities. Chicago, IL: University of Chicago Press. Thurstone, L. L. (1938b). The perceptual factor. Psychometrika, 3, 1-17. doi: 10.1007/BF02287914

Thurstone, L. L., \& Thurstone, T. G. (1941). Factorial studies on intelligence. Chicago, IL: University of Chicago Press.

Skirbekk, V., Stonawski, M., Bonsang, E., \& Staudinger, U. M. (2013). The Flynn effect and population aging. Intelligence, 41, 169-177. doi: 10.1016/j.intell.2013.02.001

Undheim, J. O., \& Gustafsson, J.-E. (1987). Hierarchical organization of cognitive abilities: Restoring general intelligence through the use of linear structural relations (LISREL). Multivariate Behavioral Research, 22, 149-171. doi: 10.1207/s15327906mbr2202_2

Valentin Kvist, A., \& Gustafsson, J.-E. (2008). The relation between fluid intelligence and the general factor as a function of cultural background: A test of Cattell's investment theory. Intelligence, 36, 422-436. doi: 10.1016/j.intell.2007.08.004

Vernon, P. A. (1988). The generality of g. Personality and Individual Differences, 10, 803-804. doi: 10.1016/0191-8869(89)90129-3

Vernon, P. E. (1950). The structure of human abilities. London: Methuen. 
Waller, N. (2007). A general method for computing hierarchical component structures by Goldberg's Bass-Ackwards method. Journal of Research in Personality, 41, 745-752. doi:10.1016/j.jrp.2006.08.005

Wothke, W., Bock, R. D., Curran, L. T., Fairbank, B. A., Augustin, J. W., Gillet, A. H. et al., (1990). A factor analytic examination of the armed services vocational aptitude battery $(A S V A B)$ and the kit of factor-referenced tests. San Antonio, TX: Brooks Air-Force Base. (AFHRL-TR-90-67). Retrieved from http://www.icodap.org/papers/AFHRL/AFHRL-TR90-67DRAFT.pdf

Yung, Y. F., Thissen, D., \& McLeod, L. D. (1999). On the relationship between the higher-order factor model and the hierarchical factor model. Psychometrika, 64, 113-128. doi: 10.1007/BF02294531

Zuckerman, M., Kuhlman, D.M., \& Camac, C. (1988). What lies beyond E and N? Factor analyses of scales believed to measure basic dimensions of personality. Journal of Personality and Social Psychology, 54, 96-107. doi: 10.1037/0022-3514.54.1.96 
Table 1

Some Common Ideas on Factor Taxonomies at Different Levels of the Intelligence Hierarchy

\begin{tabular}{ll}
\hline Factors & Model/Author(s) \\
\hline 1 & Jensen, 1998; Lubinski, 2004; P. A. Vernon, \\
& 1988 \\
1 & $\begin{array}{l}\text { Gustafsson, 1984; Jensen, 1998; Undheim \& } \\
\text { Gustaffson, 1987; Spearman, 1904 }\end{array}$ \\
1 & Robinson (1999, 2005) \\
1 & Ashton, Lee, and P. A. Vernon (2001, 2005) \\
2 & P. E. Vernon's Hierarchical Theory of \\
& Intelligence (P. E. Vernon, 1950)
\end{tabular}

2 gf-gc theory (R. B. Cattell, 1943b, 1963, J. L. Horn, 1965a, 1976 Prediction

Explains $50 \%$ of the variance

Fluid tasks like Raven's progressive matrices have the highest loadings.

Knowledge and acquired skills have the highest loadings

Fluid and crystallized show similar loadings

v:ed (verbal:educational) captures abilities like verbal ability, numerical facility, logical reasoning and fluency perceptual factor labeled k:m (spatial:mechanical) that is dominated by spatial abilities, mechanical information, psychomotor coordination, reaction times and manual skills

general crystallized factor (gc) capturing the ability to use knowledge, acquired skills, and experience as well as a general fluid factor (gf) tapping the ability to think logically and solve problems in novel situations.

3 Gustafsson's HILI model (Gustafsson, 1984)

gf-gc and a visualization factor (gv) that includes all tasks with figural content.

3 Verbal-Perceptual-Image Rotation Model

Verbal and perceptual factors similar to Vernon's hierarchical theory, additional image rotation factor

(Johnson \& Bouchard, 2005)

3 Figural-Verbal-Numerical Taxonomy (Ackerman, 1988; Ackerman, Beier, \& Boyle, 2005; Marshalek Lohman, \& Snow, 1983), also included structure of intellect model (Guilford, 1967) and the Berlin model of intelligence structure (Süß \& Beauducel, 2005)

4 Ackerman's modified version of the radex model (Ackerman, 1988; Ackerman et al., 2005)

4 Operation facets of the Berlin model of intelligence structure (Beauducel \& Kersting, 2002; Jäger, Süß, \& Beauducel, 1997; Süß \& Beauducel, 2005)

5 Extended gf-gc theory(Hakstian \& Cattell, 1974; J. L. Horn, 1965a; J. L. Horn \& Cattell, 1966)

7 Three stratum theory of cognitive abilities (Carroll, 1993)

figural, verbal, and numerical power abilities and more basic speed abilities

a broad reasoning factor that primarily captures the ability to think logically, a memory factor, a fluid ability factor that taps the ability to generate diverse ideas and material like words or figural shapes, and a speed factor that captures the ability to process material and quickly solve simple tasks.

gf-gc and additionally: general cognitive speed (gs), visualization capacity (gv) that taps the ability to integrate and organize visual material, general memory capacity (gm), and general retrieval capacity (gr) that captures the ability to retrieve information from long-term memory and is largely similar to the concept of fluency in other intelligence models. A notable difference, however, is that verbal and word fluency factors are assigned to gc and not to the gr dimension in the theory so that that gr is not perfectly identical to fluency constructs in other frameworks.

Second stratum includes gc, gf, gv, gs, and gr from the extended gf-gc theory and additionally two new factors: Auditory perception and reaction time decision speed

Word fluency, verbal comprehension, spatial visualization, number facility, associative memory, reasoning, and perceptual speed 
Table 2

Datasets Included in the Review

\begin{tabular}{|c|c|c|c|c|c|}
\hline \multirow[b]{2}{*}{ Label } & \multirow[b]{2}{*}{$\begin{array}{c}\text { Author(s) and year of } \\
\text { publication }\end{array}$} & \multicolumn{2}{|c|}{ Sample } & \multicolumn{2}{|r|}{ Material } \\
\hline & & $N$ & Characteristics & No. & Source(s) \\
\hline THUR41 & $\begin{array}{l}\text { Thurstone and } \\
\text { Thurstone (1941) }\end{array}$ & 710 & $\begin{array}{l}\text { Elementary } \\
\text { school children, }\end{array}$ & 60 & $\begin{array}{l}\text { New developments; tests } \\
\text { from previous publications by } \\
\text { L. L. Thurstone (1938a, } \\
\text { 1938b) }\end{array}$ \\
\hline HORN65 & J. L. Horn (1965a) & 297 & $\begin{array}{l}\text { Prisoners, school } \\
\text { children, and } \\
\text { unemployed } \\
\text { persons, mean } \\
\text { age: } 27.6 \text { years } \\
(S D=10.6 ; \\
\text { Range }=14-61) ; \\
27.61 \text { percent } \\
\text { female }\end{array}$ & $38^{\mathrm{b}}$ & $\begin{array}{l}7 \text { tests taken from Botzum } \\
(1951) \\
5 \text { tests taken from previous } \\
\text { research by R. B. Cattell } \\
2 \text { tests from previous research } \\
\text { by J. P. Guilford } \\
3 \text { tests from previous research } \\
\text { by L. L. Thurstone } \\
1 \text { test taken from Taylor } \\
(1947)\end{array}$ \\
\hline SCHO76 & Scholl (1976) & 276 & $\begin{array}{l}\text { German police } \\
\text { trainees, mean } \\
\text { age: } 20.4 \text { years } \\
\text { (Range }=16-36)\end{array}$ & 63 & $\begin{array}{l}57 \text { translations of tests from } \\
\text { the } 1963 \text { Kit of Factor- } \\
\text { Referenced Tests (French, } \\
\text { Ekstrom, \& Price, 1963); } \\
3 \text { newly developed tests; } \\
2 \text { tests from the } \\
\text { Leistungsprüfsystem (LPS; } \\
\text { W. Horn, 1962); } \\
1 \text { test from the } \\
\text { Intelligenzstrukturtest (I-S-T; } \\
\text { Amthauer, 1955) }\end{array}$ \\
\hline WOTH90 & $\begin{array}{l}\text { Wothke, Bock, } \\
\text { Curran, Fairbank, } \\
\text { Augustin, Gillet, } \\
\text { and Guerrero } \\
\text { (1990) }\end{array}$ & $\begin{array}{l}6,751 \text { overall but } \\
\text { participants } \\
\text { worked on } \\
\text { different booklets, } \\
N \text { s for each } \\
\text { correlation vary } \\
\text { between } 207-701 \text {; } \\
\text { harmonic mean } \\
230\end{array}$ & $\begin{array}{l}\text { Air-force recruits, } \\
16.9 \text { percent } \\
\text { female }\end{array}$ & 56 & $\begin{array}{l}\text { Armed Services Vocational } \\
\text { Aptitude Battery (ASVAB, } \\
10 \text { tests); } 46 \text { tests from the } \\
1976 \text { Kit of Factor- } \\
\text { Referenced Tests (Ekstrom, } \\
\text { French, Harman, \& Derman, } \\
\text { 1976) }\end{array}$ \\
\hline BEAU02 & $\begin{array}{l}\text { Beauducel and } \\
\text { Kersting }(2002)^{\mathrm{a}}\end{array}$ & 16,823 & $\begin{array}{l}\text { Applicants for } \\
\text { governmental } \\
\text { positions in } \\
\text { Germany, Mean } \\
\text { age: } 21.39(S D= \\
4.41 ; \text { Range }=15- \\
57) ; 58.10 \text { percent } \\
\text { female }\end{array}$ & 44 & $\begin{array}{l}\text { New developments; fluid } \\
\text { tasks are based on previous } \\
\text { work by Jäger, Süß, and } \\
\text { Beauducel (1997) }\end{array}$ \\
\hline
\end{tabular}

${ }^{\text {a }}$ The dataset we analyzed in the current article is an extended version of the dataset analyzed in the 2002 publication.

${ }^{\mathrm{b}}$ Additionally includes six tests measuring attention and one test that was highly similar to another test (both were mechanical knowledge tests and correlated at $r=.69$ ). Because attention is considered to be related to intelligence but is treated as a separate construct in the literature (e.g., de Jong \& Das-Smaal, 1995; Schweizer, Moosbrugger \& Goldhammer, 2005) and because the parallel tests yielded a strong test-specific factor, these tests were excluded. 

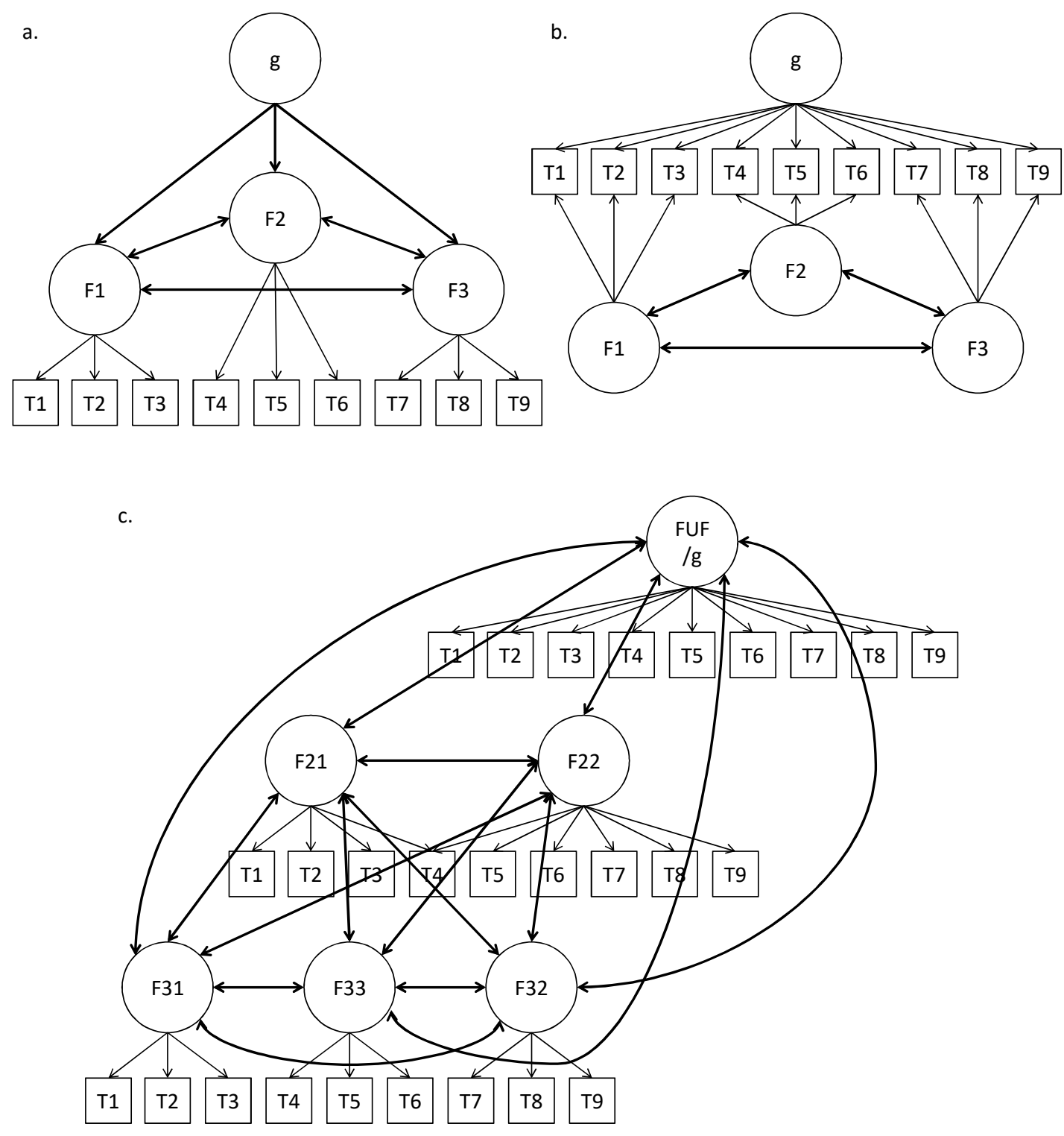

Figure 1. The higher-order model and the bi-factor model are shown in (a) and (b), respectively. These models seek to find the most parsimonious hierarchical solution. In contrast, the hierarchies of factor solutions approach shown in (c) separately extracts solutions with different numbers of factors from the same indicators and then studies correlations between these factors and represents a different analytical strategy. FUF $=$ first unrotated factor. 


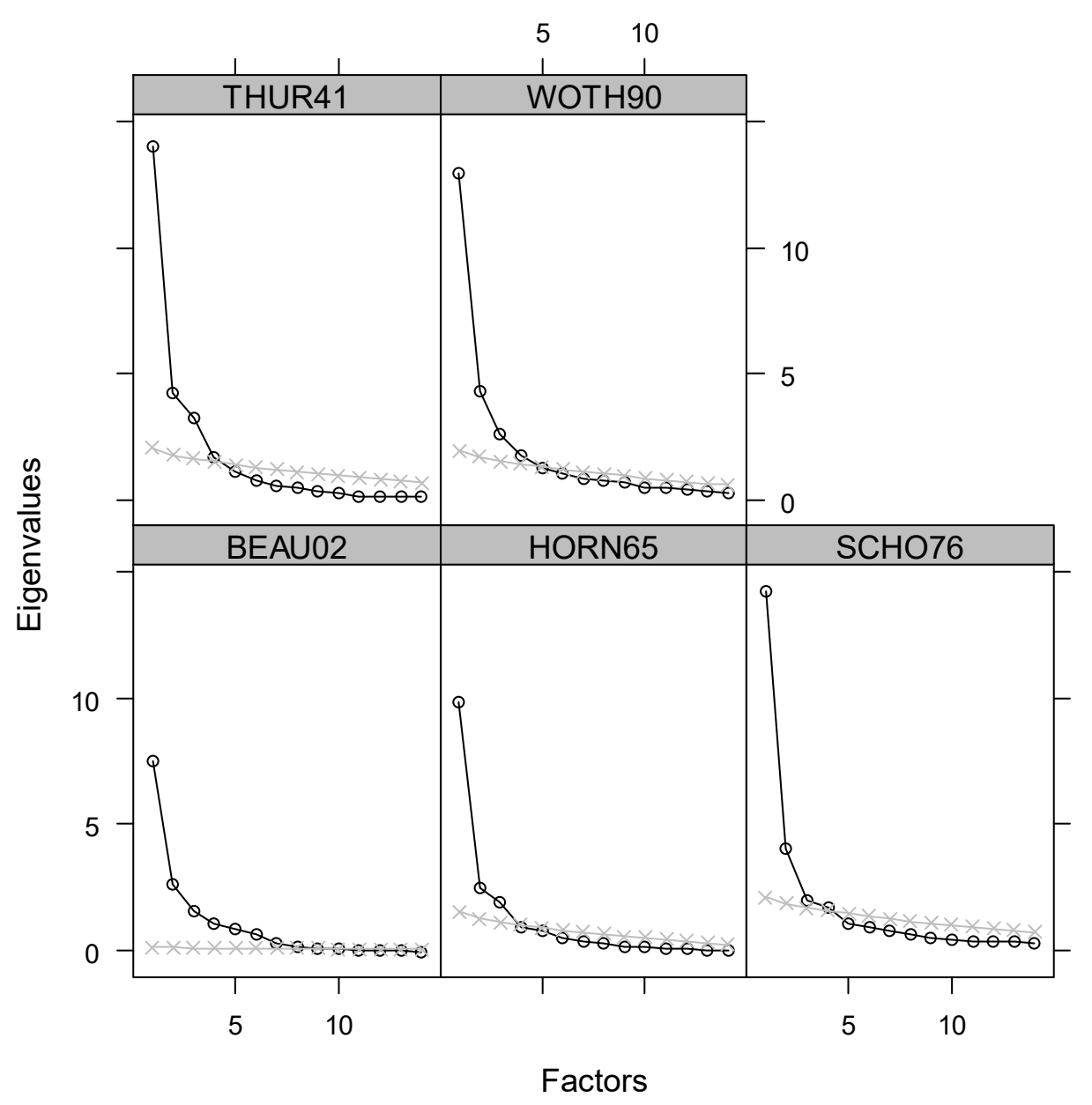

Figure 2. First fifteen eigenvalues (o) and first fifteen simulated random eigenvalues from Horn's parallel analysis procedure (x) for the five datasets included in the present study. 


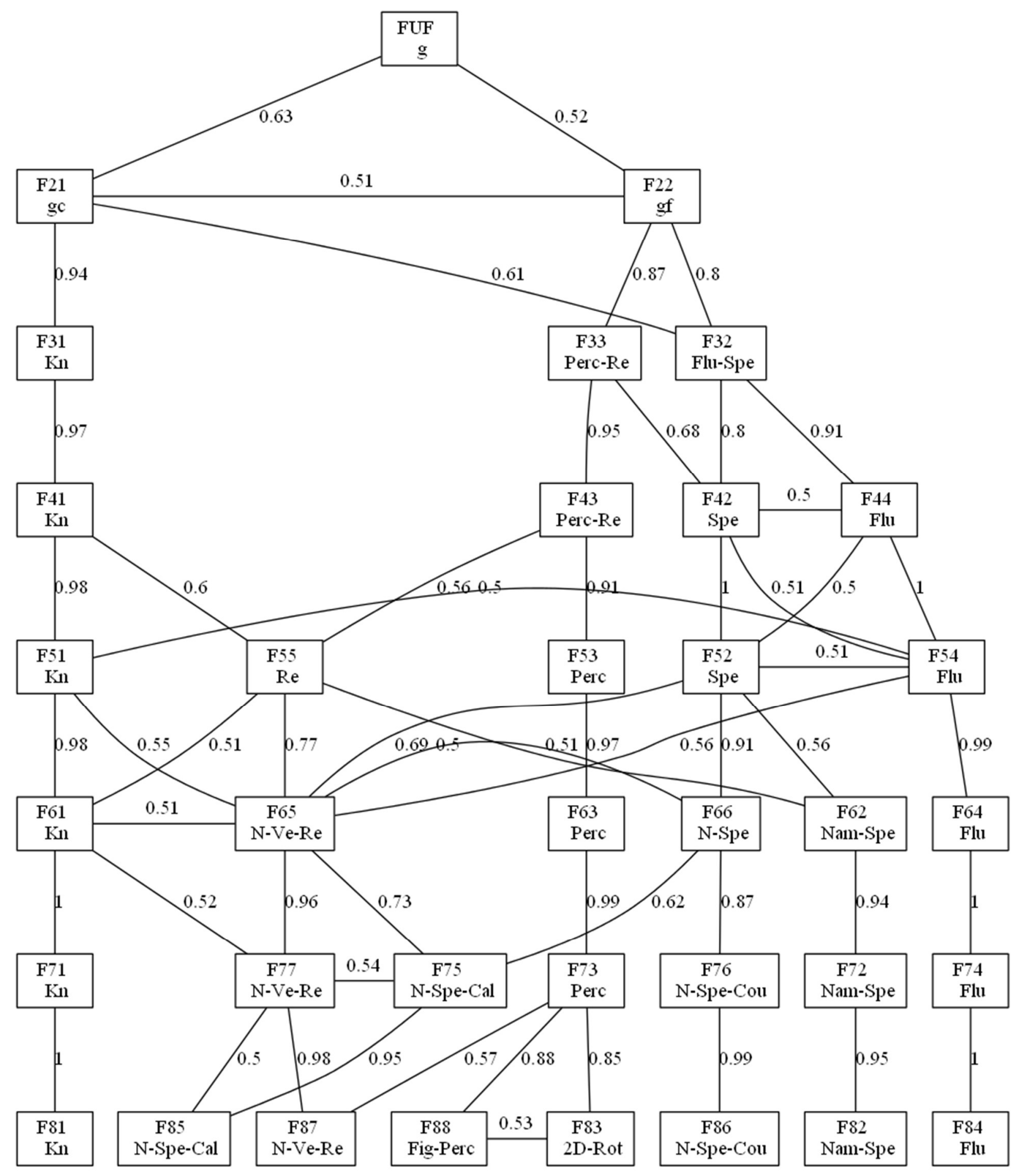

Figure 3. The hierarchical structure of the Thurstone and Thurstone (1941) dataset. 


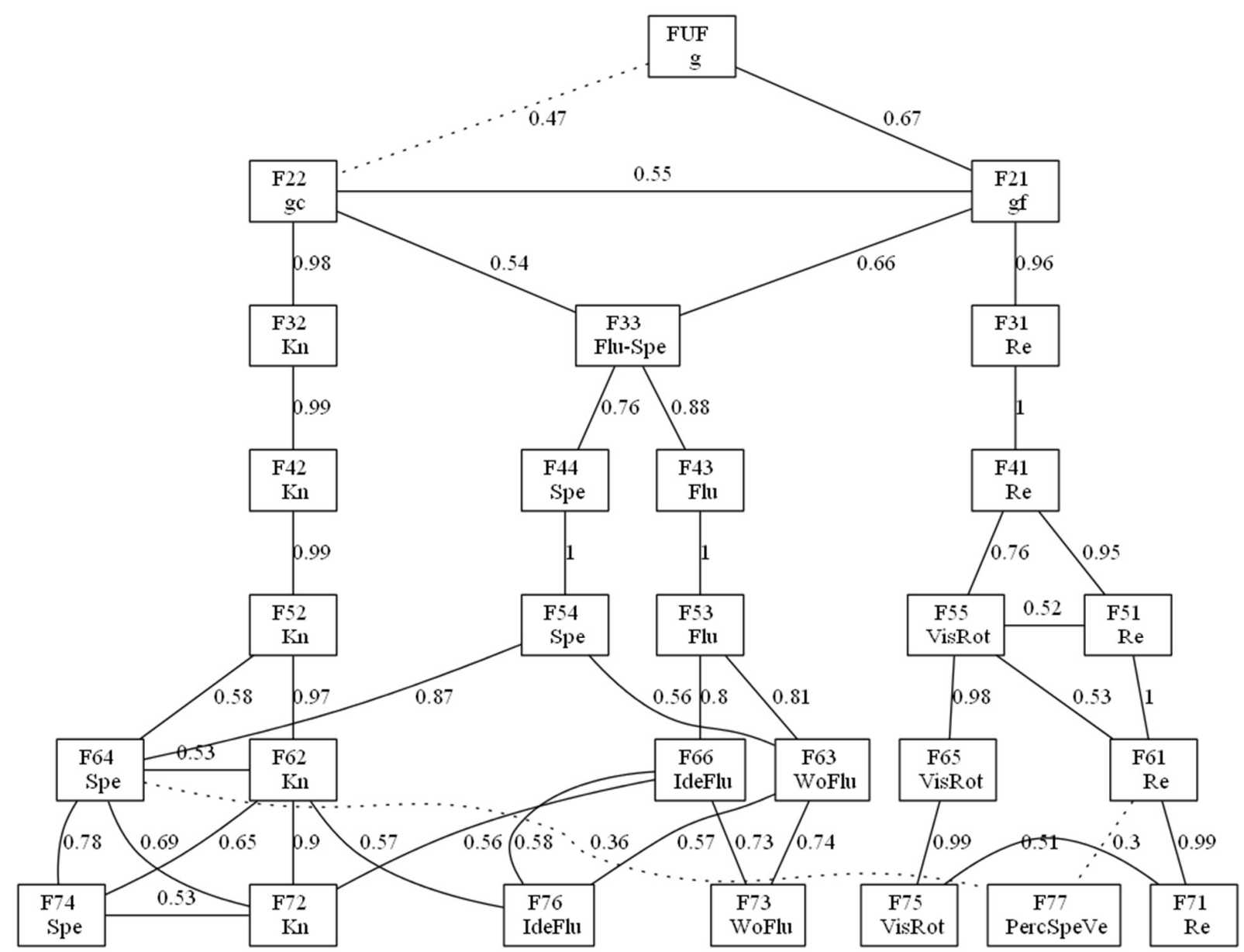

Figure 4. The hierarchical structure of the J. L. Horn (1965a) dataset. 


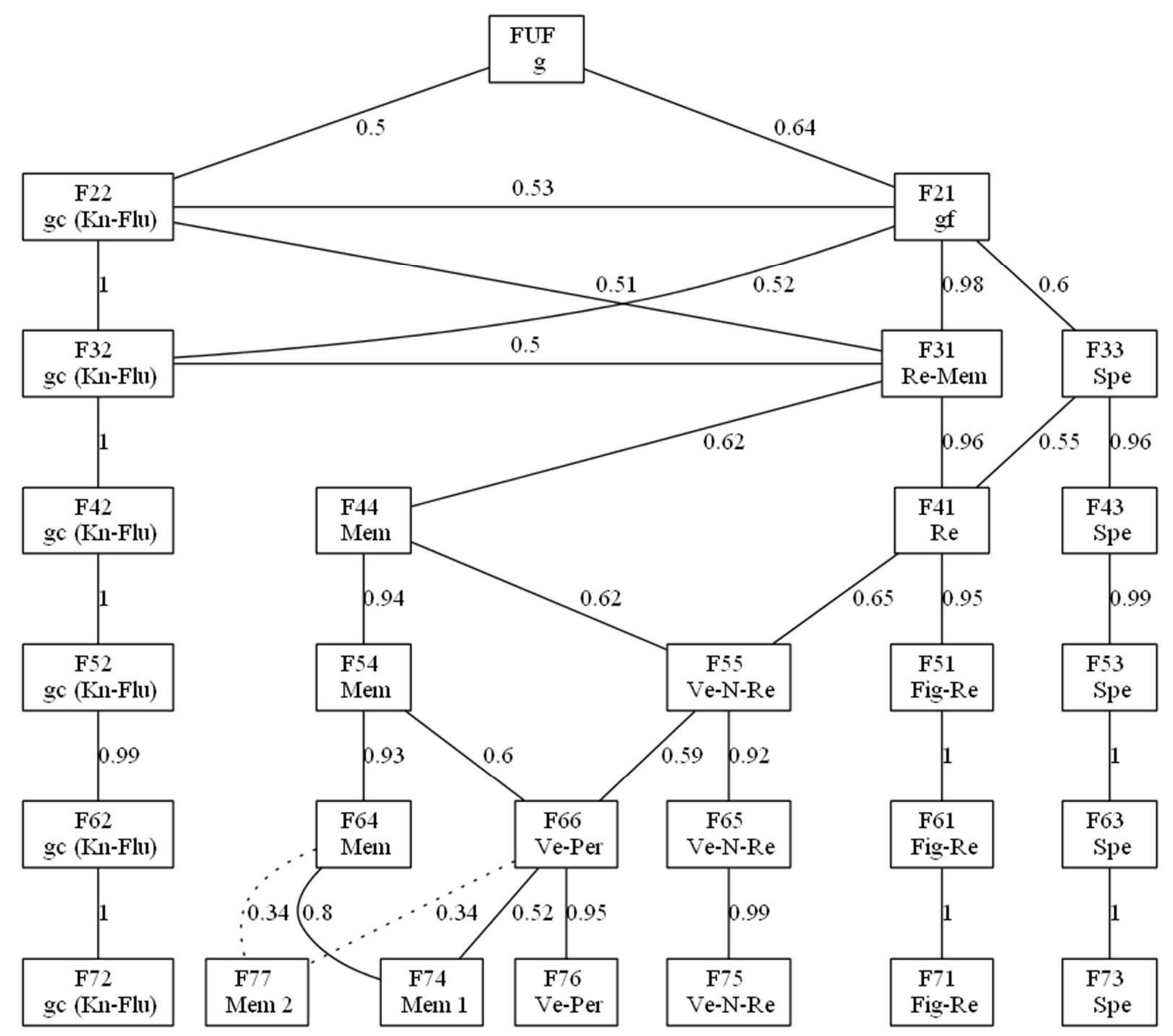

Figure 5. The hierarchical structure of the Scholl (1976) dataset. 


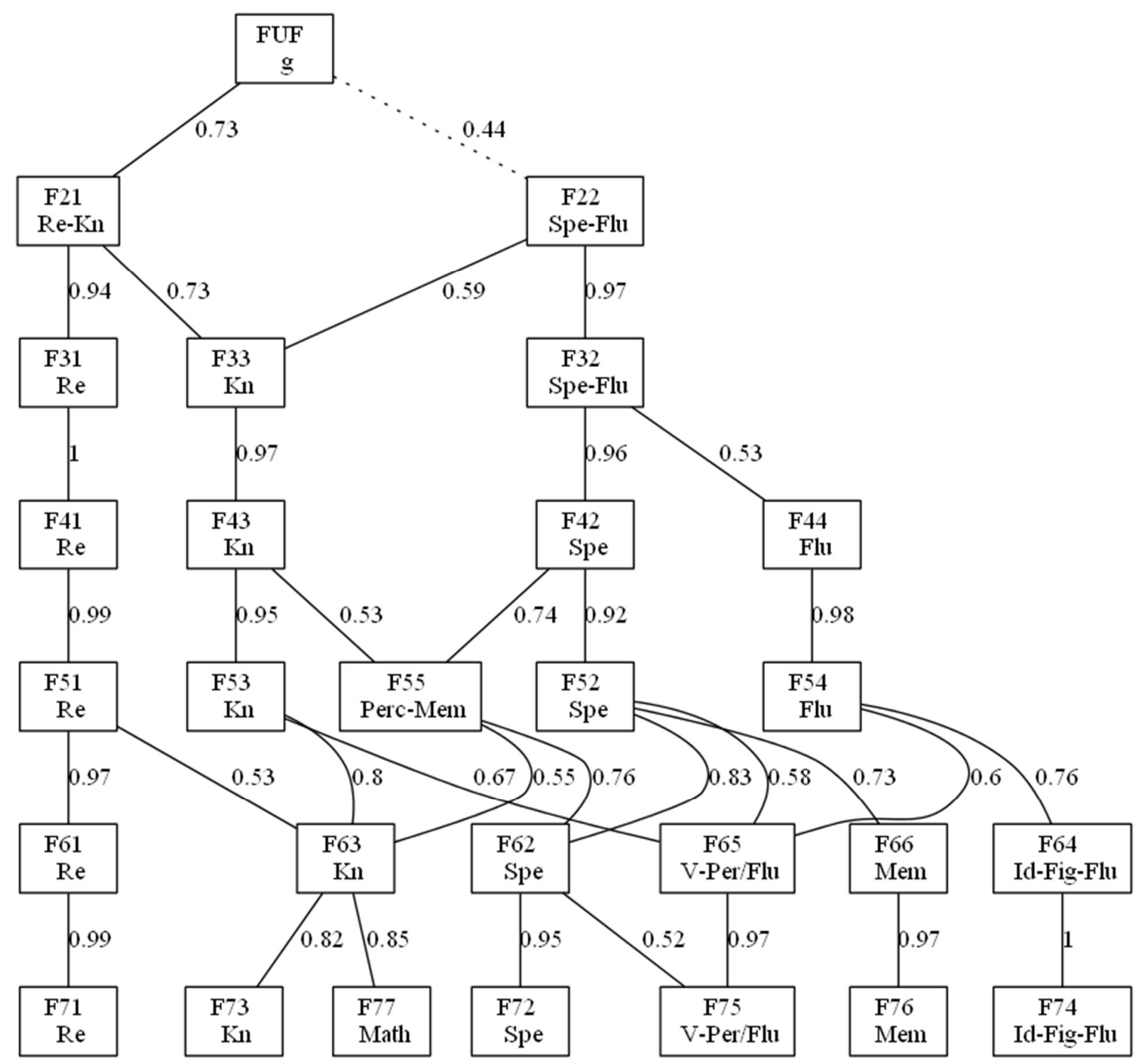

Figure 6. The hierarchical structure of the Wothke, Bock, Curran, Fairbank, Augustin, Gillet, and Guerrero (1990) dataset. 


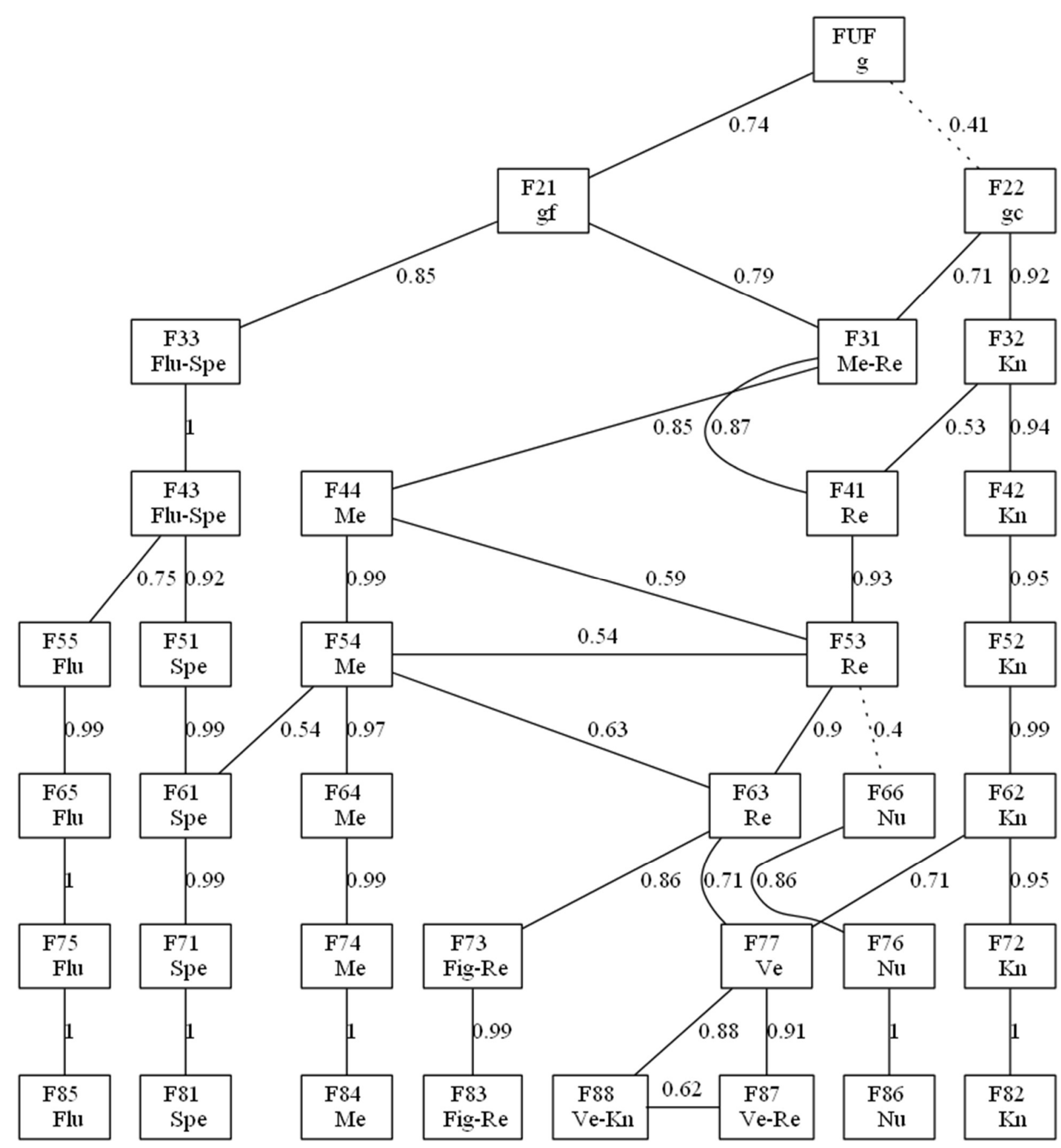

Figure 7. The hierarchical structure of the Beauducel and Kersting (2002) dataset. 


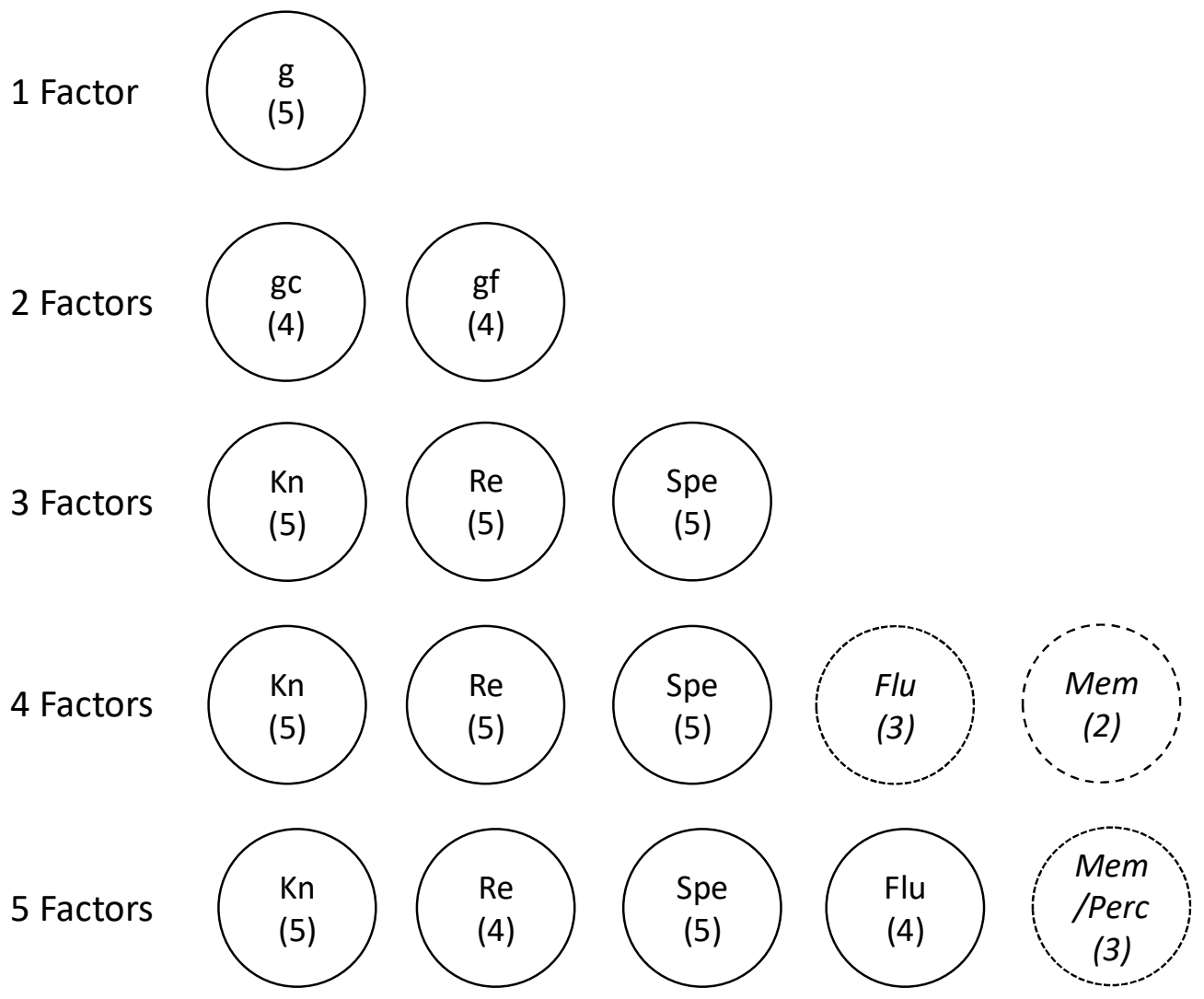

Figure 8 . Overview and summary of the findings. The numbers in parentheses indicate the number of datasets with evidence for the respective factor. 